\title{
An analysis of Pc3 and Pc4 pulsations at Terra Nova Bay (Antarctica)
}

\author{
U. Villante, M. Vellante, G. De Sanctis \\ Dipartimento di Fisica, Università and Area di Astrogeofisica. 67100. L'Aquila, Italy
}

Received: 27 December 1999 / Revised: 3 July 2000 / Accepted: 21 July 2000

\begin{abstract}
We conducted a statistical analysis of the physical characteristics of the micropulsation activity (Pc3 and Pc4 range) detected, during the austral summer 1994-95, at Terra Nova Bay (Antarctica, corrected latitude $80.0^{\circ} \mathrm{S}$ ), a station which is few degrees poleward of those where most of the Antarctic measurements in these frequency ranges have been performed. The emerging overview suggests that the correspondence between the pulsation power and the external parameters (solar wind speed, interplanetary magnetic field magnitude and orientation) is significantly stronger than at somewhat lower latitudes. The day-to-day power variability was found to be strictly related to the general level of the geomagnetic activity, and the power level sharply maximizes at local magnetic noon. In the Pc4 range peaks of correlation with the SW speed are found in the dawn and dusk sides of the Earth's magnetosphere and the daily variation of the polarization pattern is closely consistent with that found at auroral latitudes and at lower frequencies. In the Pc3 range the correlation coefficient between the pulsation power and the SW speed has maximum values in the local morning, and the frequency of selected events reveals a strong IMF control during closed magnetospheric conditions. The local time dependence of the correlation coefficient between the pulsation power and the cone angle reveals an additional control by the IMF orientation, which becomes more explicit around local noon.
\end{abstract}

Key words: Magnetospheric physics (magnetopause, cusp, and boundary layers)

\section{Introduction}

As underlined by several authors, geomagnetic pulsation research at high latitudes is important in that local field

Correspondence to: U. Villante

e-mail: umberto.villante@aquila.infn.it lines penetrate extreme magnetospheric regions where several generation mechanisms for ULF waves are active (reviews by Fraser-Smith, 1982; Hughes, 1982; Arnoldy et al., 1988). In these regions, Pc3 (10-45 s) and Pc4 pulsations (45-150 s) typically reach maximum amplitudes at geomagnetic latitudes of $\sim 70^{\circ}$ and appear to decline rapidly as the point of observation moves away from the polar cap boundary toward the geomagnetic poles (Fraser-Smith, 1982; Engebretson et al., 1986). In particular, Bolshakova and Troitskaya (1984) found that the maximum amplitude of Pc3 pulsations coincides with the position of the dayside cusp to within $5^{\circ}$ to $8^{\circ}$, and varies in latitude with cusp position during magnetic disturbances. Moreover, Troitskaya (1985) showed that the Pc3 spectrum displays a maximum near the cusp and suggested that the cusp might be tracked using Pc3 pulsations. At lower frequencies (120-600 s), Vennerstrom (1999) recently examined the dayside magnetic variations at a number of stations in Greenland, Scandinavia and Canada (invariant latitudes between $67^{\circ}$ and $86^{\circ}$ ) and found maximum amplitudes between $70^{\circ}$ and $75^{\circ}$. On the other hand, Engebretson et al. (1989) conducted a comparison between South Pole (corrected geomagnetic latitude $74.2^{\circ}$ ) and McMurdo observations $\left(80^{\circ}\right)$ to delineate significant morphological differences between selected Pc3 events and more irregular Pil events (1-40 s): they found in particular that the Pil activity, which is important at South Pole, is weak at McMurdo, and its occurrence might be related to the southward turning of the IMF orientation.

The occurrence properties of Pc3 and Pc4 pulsations in the polar caps could be considered similar to those observed at lower latitudes in that these fluctuations appear to represent predominantly a daylight phenomenon which becomes more frequent in the local summer (Fraser-Smith, 1982). Wolfe et al. (1987), who examined the power level of pulsations ( $H$ component, JuneSeptember, 1982) in the Pc3 and Pc4 band at South Pole, found in both frequency bands that largest power enhancements were one to two hours before the local magnetic noon. Similar results were obtained from the same station (May-August, 1990) by Engebretson et al. 
(1994), who examined optical and magnetic pulsations in the time interval 0700-1600 MLT and concluded that both kinds of pulsations showed a maximum occurrence somewhat before local noon for all levels of geomagnetic activity. Morris and Cole (1987) found at Davis $\left(74.6^{\circ} \mathrm{S}\right.$, magnetic noon $\sim 0945$ UT), that the Pc3 occurrence (February, 1981-March, 1982) maximize between 6 and 10 UT, which might correspond to the probable location of Davis under the cusp projection. On the other hand, Engebretson et al. (1986), extended the analysis of South Pole observations to nighttime intervals for all of 1983 and found also evidence for a secondary maximum of the wave activity (1-80 s) between 2100 and 0200 MLT. Some correspondence for similar results in the Northern hemisphere was provided by Olson (1986), who examined Cape Parry observations $\left(74.8^{\circ} \mathrm{N}, 273\right.$ days of data during 1983) and found a broad band increase in the ULF spectra (Pc3-Pc5) around magnetic noon (with maximum values typically encountered before noon) and a minor amplitude maximum in the nighttime hemisphere, in connection with substorms. At lower frequencies (180-420 s), Troitskaya (1985) found, at latitudes of $\sim 78^{\circ}$, diurnal variations of irregular pulsations maximized and symmetric around magnetic noon, while Vennerstrom (1999) estimated a prominent maximum in the morning side and a secondary maximum, related to substorms, in the nightside.

The occurrence rate and the amplitude of micropulsation events at high latitudes are both significantly influenced by the solar wind (SW) speed. Wolfe et al. (1987) evaluated the linear correlation coefficient between the Pc3-Pc4 power and the SW speed for each hour of the local time and found, at South Pole, peak values of the order of 0.6 near local magnetic noon and approximately four hours later. At a conjugate station and at lower frequencies (120-240 s), Vennestrom (1999) found a correlation coefficient of the order of 0.78 between the logarithm of the power of pulsations (6-10 MLT) and the SW speed. Engebretson et al. (1998) examined, for the last six months of 1993, the total power of the horizontal components (Pc5 band) in the auroral zone and at near cusp latitudes in the Arctic Canada and found (Kevo, corrected geomagnetic latitude $66.2^{\circ} \mathrm{N}, \quad 6-12 \mathrm{MLT}$; Cape Dorset, $74.5^{\circ} \mathrm{N}$, 8-14 MLT) correlation coefficients with the SW speed (log-log scale) of the order of $0.73-0.74$.

The interplanetary magnetic field (IMF) control of high-latitude pulsations has been investigated in several papers with some controversial results. Indeed, while the statistical analysis of South Pole data in the Pc3-Pc4 range (Wolfe et al., 1987; Yumoto et al., 1987) concluded that there is poor (or no) correlation between the power of pulsations and the cone angle (i.e. the angle between the IMF lines and the radial direction), Engebretson et al. (1986), who selected narrow band, well-identified Pc3 events found that near local noon their occurrence was strongly correlated with the radial IMF component. A similar conclusion was obtained by Engebretson et al. (1989), who identified a cone angle control on the simultaneous occurrence of narrow band dayside Pc3 pulsations at South Pole, McMurdo, and at AMPTE CEE in the dayside equatorial magnetosphere.

As remarked by several authors, there are two principal candidates for the exogenic source of highlatitude Pc3 and Pc4 pulsations: the Kelvin-Helmoltz instability (KHI) at the magnetopause boundary, and upstream waves excited by reflected ion beams in the Earth's foreshock. In this sense, the correlation between the pulsation power and the SW speed suggests that KHI plays an important role (which might be more significant at lower frequencies) both in the generation of surface waves and in the amplification of already existing waves as they are convected and transmitted through the dayside high latitude magnetopause (e.g. Yumoto et al., 1987). On the other hand, the strong correspondence between the pulsation occurrence and the radial IMF component and the relation between the pulsation frequency and the IMF strength led other authors (e.g. Engebretson et al., 1986) to suggest that upstream wave energy in the Pc3 range can reach low altitudes on cusp field lines although the KHI is likely to play an important role in amplifying existing waves as they pass through the magnetosheath. In addition, Engebretson et al. (1990) also proposed, by an analysis of a wide set of experimental observations at South Pole and McMurdo, that precipitating electrons at cleft latitudes may also be important (via modification of ionospheric currents and conductivities) in the transmission of upstream signals as well as in the local generation of dayside high-latitude pulsations in the Pc3 frequency range. On the other hand, the results obtained by Olson and Szuberla (1997) and Szuberla et al. (1998) confirmed the local aspect of the observed Pc3 pulsations in that they estimated an upper limit of the order of $140-200 \mathrm{~km}$ for the interstation coherence approximately at cusp latitudes.

In the present work we conduct a statistical analysis of the physical characteristics of the micropulsation activity (Pc3 and Pc4 range) detected, during the austral summer 1994-95, at Terra Nova Bay (TNB, Antarctica, corrected latitude $80.0^{\circ} \mathrm{S}, \quad \mathrm{MLT}=\mathrm{UT}-8 ; \quad \mathrm{LT}=$ UT +13 ), a station which is few degrees poleward of those where most of the Antarctic measurements in these frequency ranges were made. We examined the MLT dependence of the pulsation power and its relation with the external SW and IMF parameters to provide additional insights into the exogenic sources of polar cap fluctuations; in addition, we carefully examined the polarization pattern which is important for a better understanding of the propagation aspects. When compared with those obtained at other high-latitude stations, our results show that the nighttime power enhancement of the pulsation power appears confined to narrow latitudinal bands; on the other hand, the relationships with the SW and IMF parameters (as well as the observed polarization pattern) appear more explicit than at lower latitudes and reveal some features which are useful to distinguish between different generation processes.

For a discussion of the results obtained in the whole ULF band from TNB observations over the last several 
years the reader is referred to the review paper by Villante et al. (1999b).

\section{The results of a statistical analysis}

Power spectra of the horizontal geomagnetic field components (search coil measurements originally sampled at $10 \mathrm{~Hz}$ and then averaged and stored at $1 \mathrm{~Hz}$ ) were computed over consecutive $4 \mathrm{~min}$ intervals in the frequency range $4-100 \mathrm{mHz}$ by using the maximum entropy method with an autoregression order equal to 20 (Vellante and Villante, 1984). In the present work we examined data for the time interval November 21, 1994 to February 16, 1995. Hourly average values of the SW parameters for the period of interest have been obtained by WIND observations. Obviously, TNB has a variable distance, through the day, from the cusp projection and approaches closed field lines around the local magnetic noon.

\subsection{The long-term and the daily variation}

A preliminary visual inspection of the experimental measurements revealed the occurrence at TNB of irregular Pil events. These phenomena seem to occur preferentially during daytime hours, have typical amplitudes remarkably smaller than regular Pc3 and provide a significant contribution to the average power spectrum during highly perturbed days when they may persist for several hours. For comparison, almost monochromatic Pc3 pulsations, which occur at least for $60 \%$ of available days, have typical duration between 30 and $60 \mathrm{~min}$, dominate by far the power spectrum and are also detected, although with much minor amplitude, in the nighttime sector. A similar result was obtained by Engebretson et al. (1989), who concluded that Pc3 signals are clearly evident even in the predawn sector in the polar cap.

The day-to-day variability of the geomagnetic power at TNB is strongly related to the $K p$ index: this aspect is made clear in Fig. 1, where the daily values of the logarithm of the total power of components $\left(\log P_{T}\right)$ approximately in the Pc3 $(22-100 \mathrm{mHz})$ and Pc4 (6$22 \mathrm{mHz}$ ) band is compared with the daily average value of the planetary activity index. As can be seen, high $K p$ values correspond to high power levels and correlation coefficients such as 0.84 and 0.82 are estimated in the higher and lower frequency band, respectively. Likely, this feature simply reflects the close relation of both the pulsation power and the geomagnetic activity with the SW speed. We evaluated, indeed, the correlation coefficient between $K p$ and the SW speed and found $r=0.7$ in the period of interest. On the other hand, the pulsation energy has also been found to be dramatically influenced by the SW speed (Fig. 2): we found, indeed, correlation coefficients such as 0.88 and $0.84(\mathrm{Pc} 3$ and Pc4 band) between $\log P_{T}$ and SW speed (daily average values). More particularly, a log-log approximation of the experimental data allows estimating a power law variation of the pulsation power with the SW speed such as $V^{4.2}$ and $V^{3.1}$, in the Pc3 and Pc4 band, respectively.

The daily distribution of the hourly average values of $\log P_{H}, \log P_{D}$ (Fig. 3) reveals, for both components, a daily variation with a clear maximum around magnetic noon (with a total excursion of an order of magnitude, more explicit in the $H$ component), while it does not show any evidence for the secondary maximum detected at other high latitude stations at local midnight (Engebretson et al., 1986; Morris and Cole, 1986).
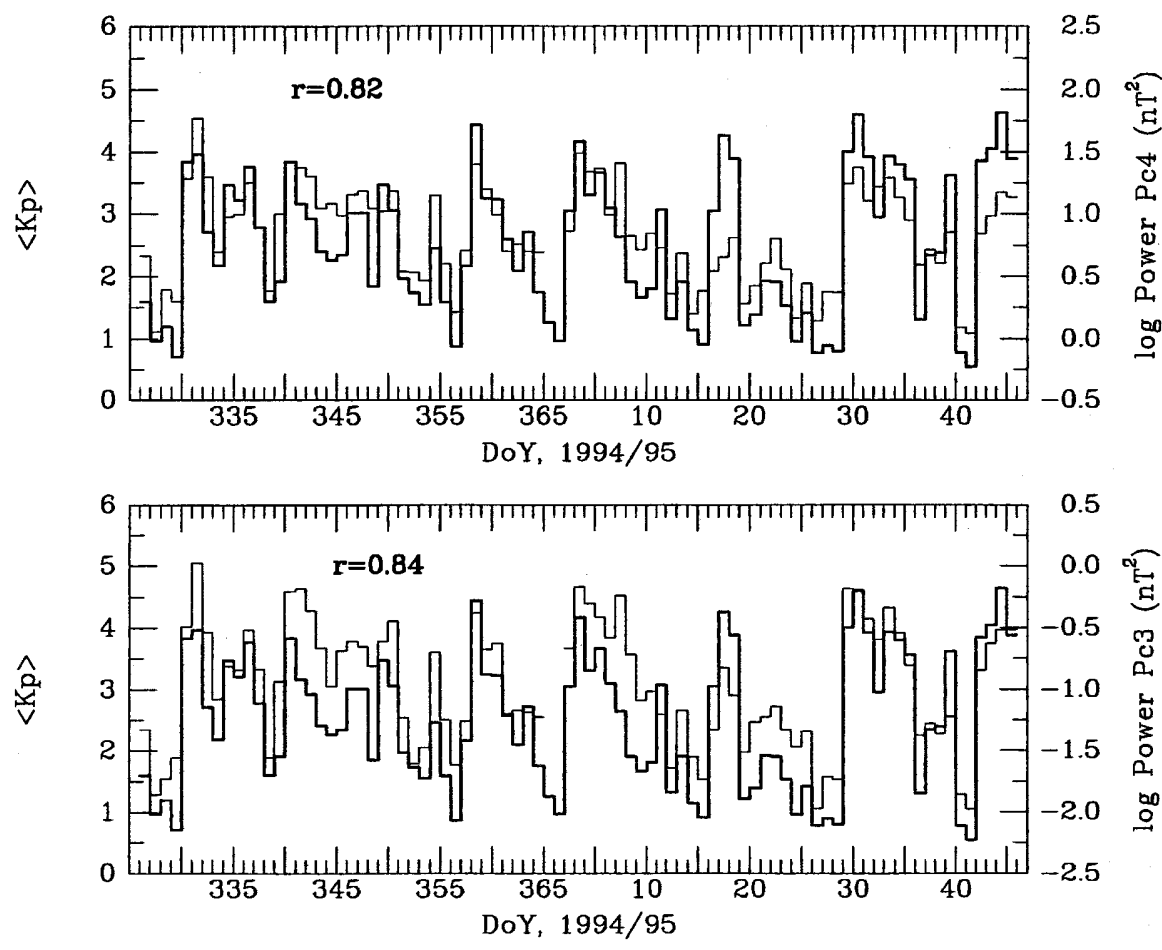

Fig. 1. A comparison between the daily average values of the $K p$ parameter (thick line) and the daily average values of logarithm of the total power of the geomagnetic field fluctuations (thin line) in the Pc3 (bottom plot) and Pc4 band 

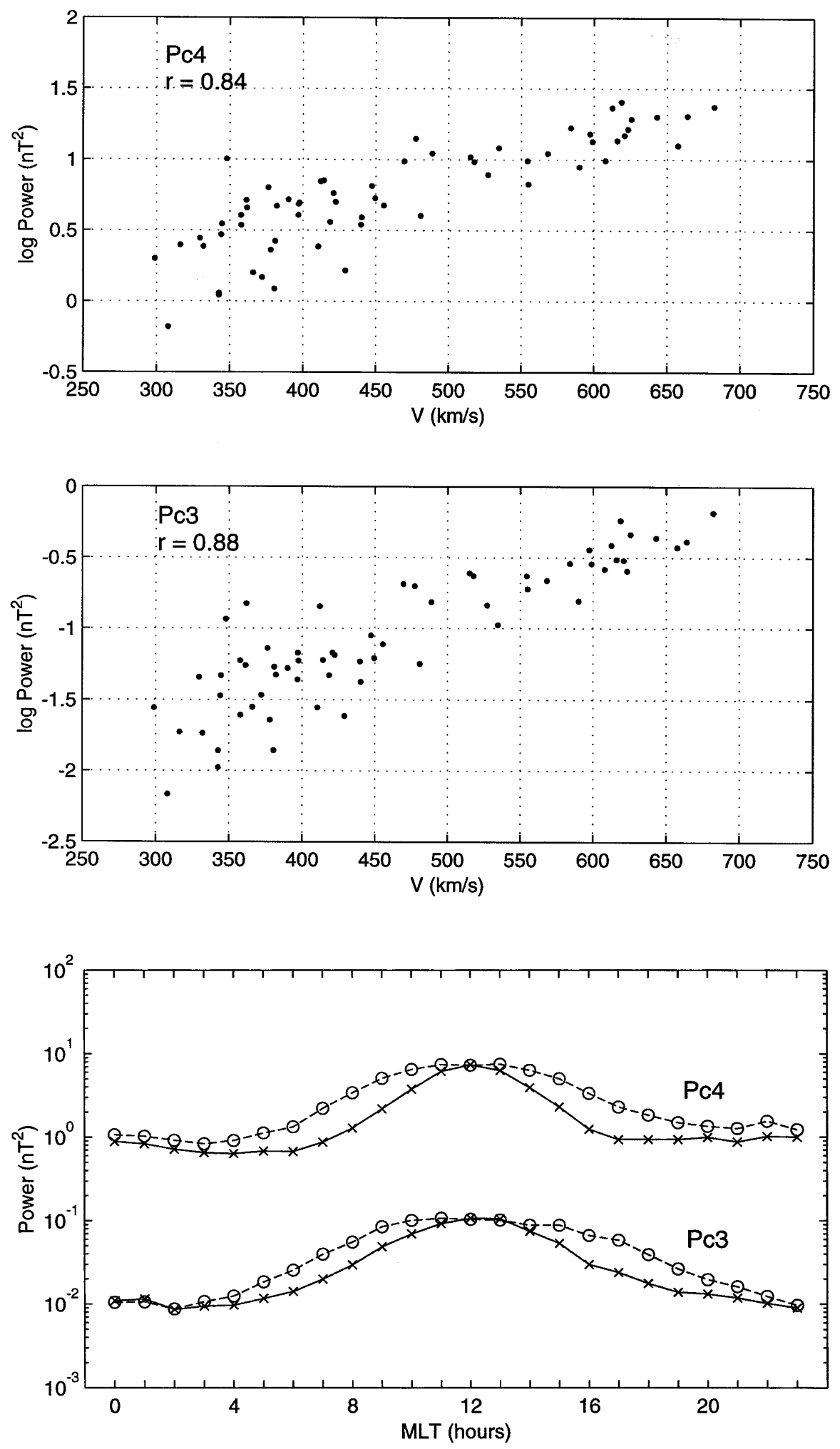

Fig. 2. The scatter plot between the logarithm of the daily average values of the total power and the daily average values of the SW speed (Pc3 band, bottom plot; Pc4 band, top plot)
Fig. 3. The daily distribution of the power of the $H$ (solid line) and $D$ (dashed line) component in the Pc3 and Pc4 band

\subsection{The MLT dependence of the SW speed control}

As previously remarked, the correlation between the pulsation power and the SW speed in general suggests an important role of the KHI instability in the generation, as well as amplification, of the magnetospheric fluctuations. To investigate this aspect better, we examined in Fig. 4 the daily variation of the correlation coefficient between $\log P_{T}$ and the SW velocity (separate analysis for each component provide approximately the same results). In this analysis the propagation time, less than $1 \mathrm{~h}$, from the variable WIND position to the magnetosphere has not been considered. On the other hand the SW speed (which typically shows significant 

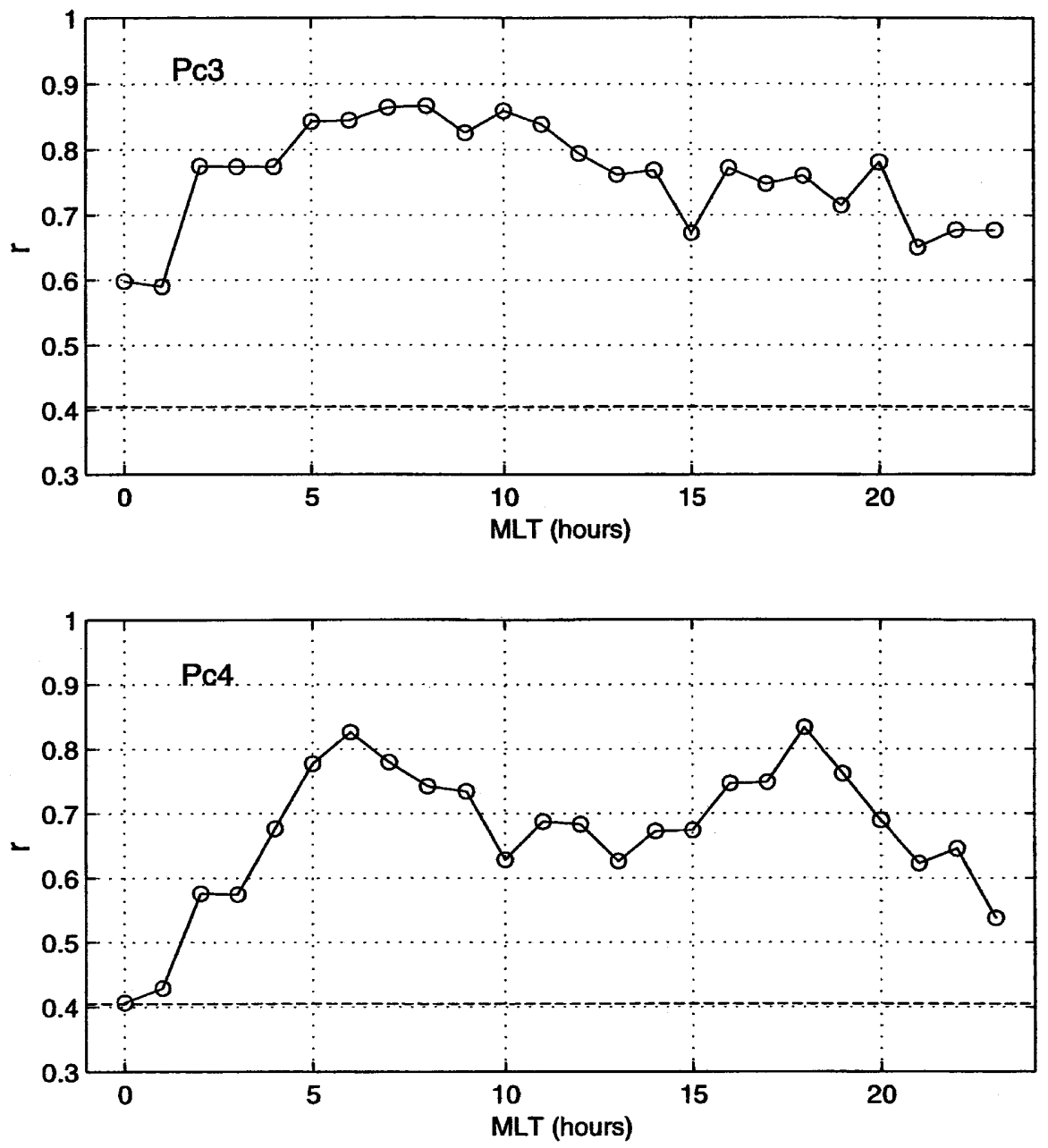

Fig. 4. The daily variation of the correlation coefficient between the logarithm of the total power and the SW speed for the Pc3 (top plot) and Pc4 band (bottom plot). The number of data points in each hour ranges between 62 and 66. Dashed lines correspond to the $99.9 \%$ confidence level variations on much larger time scales (of the order of 1-2 days) in the period of interest has an autocorrelation coefficient equal to 0.994 at a lag time of $1 \mathrm{~h}$. As shown in Fig. 4 (top plot), in the Pc3 band, the correlation coefficient is high for the whole day: in particular, it attains greater values in the local morning ( $r>0.8$ between 05-11 MLT); then it shows a general trend for a progressive decrease up to minimum night values of the order of 0.6 (i.e. still well above the $99.9 \%$ confidence limit, $r \sim 0.4$ ). Separate analysis for narrower frequency bands (not shown) suggest a similar behaviour (i.e. higher $r$ values in the morning than in the afternoon hours) in the entire Pc3 band: however, $r$ progressively decreases with the increasing frequency: for example, its morning side maximum is $\sim 0.9$ at $22 \mathrm{mHz}$ and decreases to $\sim 0.76$ at $100 \mathrm{mHz}$.

The correlation coefficient between $\log P_{T}$ and the SW speed is generally high also in the Pc4 band (minimum midnight values are now of the order of 0.4 , Fig. 4, bottom plot): moreover, it shows two prominent peak values $(r \sim 0.85)$ at the magnetic dawn and dusk meridians, while in the wide noon sector $(10-15$ MLT) it decreases to average values of the order of 0.65. In this case separate analysis (not shown) conducted for narrower frequency bands show that the morning and afternoon maxima of comparable height more clearly emerge at the lowest frequencies $(<12 \mathrm{mHz}$ ), while at higher frequencies the $r$ behaviour progressively approaches that one observed in the Pc3 band.

\subsection{The IMF control}

In order to investigate the possible influence of penetration within the high-latitude magnetosphere of upstream wave phenomena, we also conducted a correlation analysis of the Pc3 and Pc4 power with the cone angle, $\theta_{B X}$. In this case, since the IMF direction may have significant variations on time scales smaller than $1 \mathrm{~h}$, we restricted our analysis to the time interval in which the propagation time from the WIND position to the Earth's magnetosphere was smaller than $20 \mathrm{~min}$ and did not take into account the effects of the time lag. In addition, in order to increase the statistical significance, we evaluated the daily variation of the correlation coefficient on time intervals of $3 \mathrm{~h}$. The results of Fig. 5 reveal that the power of pulsations is also influenced by the cone angle, with correlation coefficients which are more significant in the Pc3 range and in the wide dayside sector, and attain highest values around local noon. 

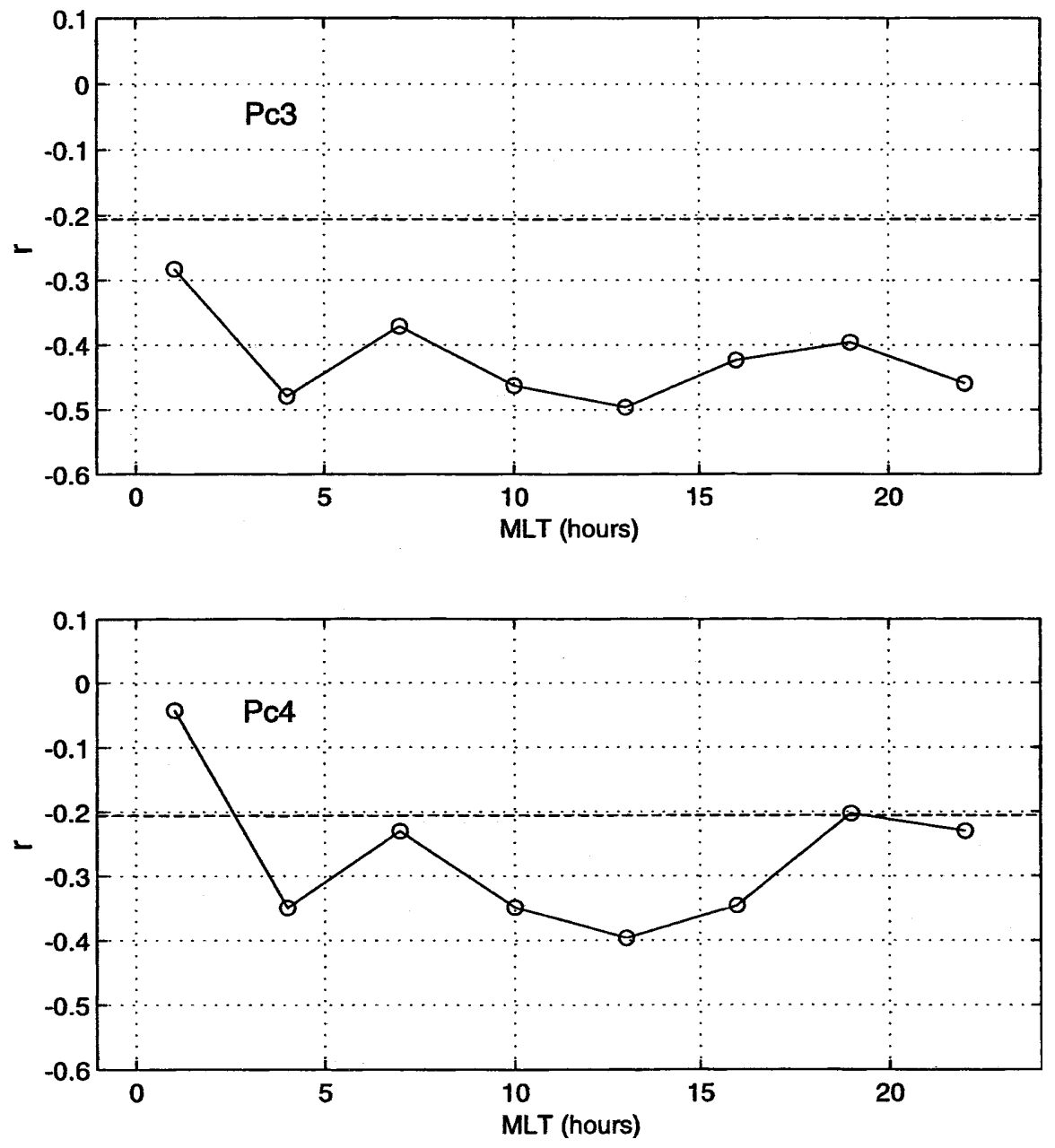

Fig. 5. The daily variation of the correlation coefficient between the logarithm of the total power and the angle between the IMF and the radial direction for the Pc3 (top plot) and Pc4 band (bottom plot). Dashed lines correspond to the $95 \%$ confidence level
As is well known, a close relationship between the frequency of pulsation events and the IMF strength [f $(\mathrm{mHz}) \sim 6 \mathrm{~B}(\mathrm{nT})$, Troitskaya and Bolshakova, 1988] has been detected in a wide latitudinal range, although some controversial results have been obtained at high latitudes. In Fig. 6 we show the results obtained for few daytime events. These events are initially selected in the individual 4 min spectra when the power at the peak frequency, $f_{\max }$, overcomes by a given threshold $T 1$ the average power evaluated over a constant amplitude (log scale) frequency interval centred in $f_{\text {max }}$; then, the power of selected events is requested to overcome by a threshold $T 2$ the power at the same $f_{\max }$ frequency in the reference spectrum (Vellante et al., 1989) evaluated for the whole time interval examined in the present investigation. As discussed by De Sanctis (1998) a definite choice of $T 1$ and $T 2$ allows us to select the most energetic, monochromatic events in the period of

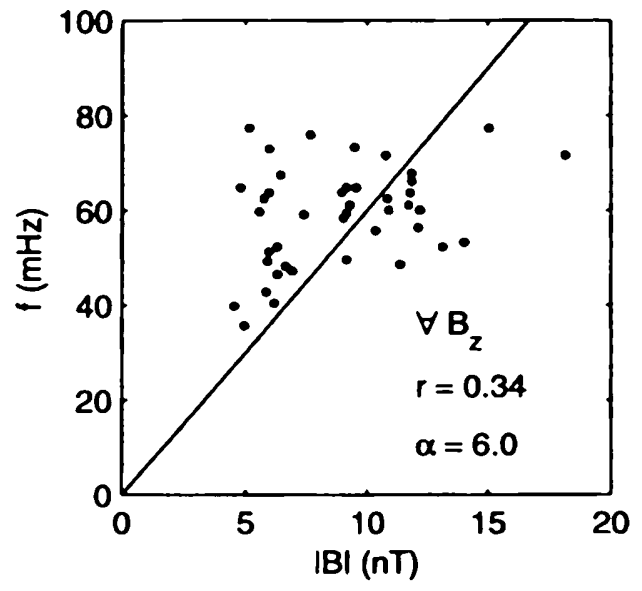

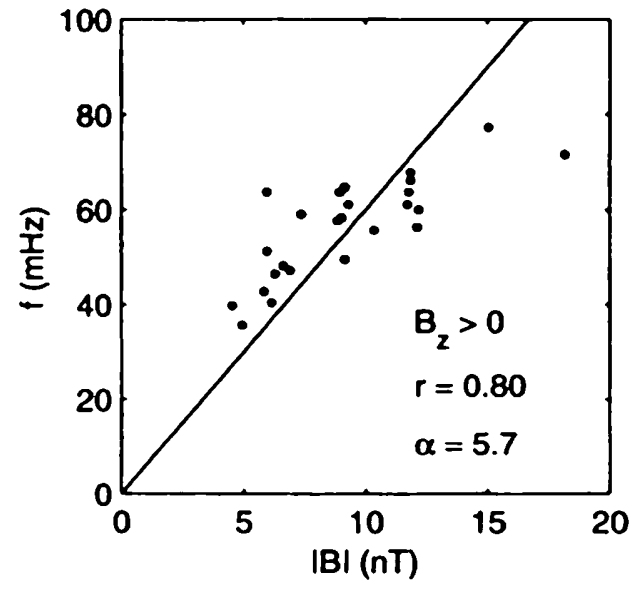

Fig. 6. The correspondence between the frequency of selected daytime events and the IMF strength for all events (left) and for those associated with northward IMF orientation (right).

The solid line corresponds to the empirical relationship 
interest. As shown in Fig. 6, the IMF control on the pulsation frequency appears poor $(r=0.34)$, when the whole set of selected events is considered, but becomes strict ( $r=0.8$, and the regression coefficient is 5.7) when the IMF has a northward component.

\subsection{The results of a polarization analysis}

We also performed a polarization analysis with interesting results. We examined, indeed, the cross-power spectra of the horizontal components using the technique for partially polarized waves as proposed by Fowler et al. (1967). Individual spectra were evaluated over $256 \mathrm{~s}$ intervals and the cross-spectral estimates were computed for 26 equally spaced frequencies between 4 and $102 \mathrm{mHz}$. In particular, we analyzed consecutive $30 \mathrm{~min}$ intervals with a step size of $15 \mathrm{~min}$. Then, for each $30 \mathrm{~min}$ interval the polarization ratio $R$ (i.e. the ratio between the polarized and total intensity of the horizontal signal) and the ellipticity $E$ (i.e. the ratio between the minor and the major axis of the polarization ellipse in the horizontal plane) were computed with 14 degrees of freedom. In order to determine the confidence limits of our analysis, we preliminarily applied the method to two independent time series of more than 100000 random numbers and by processing them with the same technique adopted for the real case we obtained $R>0.7(0.75)$ for less than $3.7 \%(1.7 \%)$ of the cross-spectral estimates.

Figure 7a shows the daily behavior of the percentage of 30 min intervals having $R>0.7$ (similar results are obtained for higher $R$ values and after removing intervals in which the sense of polarization was less clearly defined, i.e. $-0.1<E<0.1$ ). As can be seen, this percentage typically ranges between $25 \%$ and $50 \%$ and shows two maxima respectively at $09-10$ MLT (more explicit at the lowest frequencies) and in the late afternoon. The results obtained for the polarization pattern are shown in Fig. $7 \mathrm{~b}$, in which bottom lines represent, for comparison, the polarization pattern inferred from the results obtained by Samson (1972) for auroral Pc5 pulsations. As can be seen, at the lowest frequencies, the experimental results show a close correspondence between the expected (for waves propagating westward in the morning and eastward in the afternoon side of the Earth's magnetosphere, when resonance effects are taken into account, Samson, 1972; Chen and Hasegawa, 1974) and the observed polarization pattern. There are four reversals of the dominant polarization sense which is clockwise $(\mathrm{CW}$, viewed in the direction of the ambient magnetic field) approximately between 23-6 MLT, counterclockwise (CCW) between 7-11 MLT, newly CW between 12-16 (with extreme percentages of CW polarization at 14 MLT), then newly CCW between 18 and 21 MLT. The polarization pattern becomes less clear with increasing frequency: however, in the Pc3 band, a definite CCW polarization still emerges in the morning sector (413 MLT, between $20-51 \mathrm{mHz}, 4-10$ MLT, at higher frequencies).

\section{Summary}

In the present work we examined some statistical aspects of the micropulsation activity ( $\mathrm{Pc} 3$ and $\mathrm{Pc} 4$ range) detected during the austral summer 1994-95 at TNB $\left(80^{\circ} \mathrm{S}\right)$, a station which is few degrees poleward of those where most of the Antarctic measurements in these frequency ranges were performed. In particular, the principal results of the present investigation can be summarized as follows:

a. The day-to-day variability of the geomagnetic power is closely related to the general level of geomagnetic activity, with correlation coefficients greater than 0.8 . The total pulsation power shows, on a daily time scale, a power law variation with the SW speed such as $V^{4.2}$ and $V^{3.1}$ in the Pc3 and Pc4 band, respectively, with correlation coefficients such as 0.88 and 0.84 .

b. The micropulsation activity exhibits a sharp daily modulation that provides much higher values around local magnetic noon, with a total excursion approximately of an order of magnitude in both frequency bands and components. Conversely, we did not find any statistical power enhancement in the nighttime sector.

c. In the Pc4 range the correlation coefficient between the pulsation power and the SW speed peaks at the magnetic dawn and dusk meridians, and the MLT dependent polarization pattern shows four reversals of the dominant polarization sense.

d. An additional control of the cone angle on the pulsation power is detected in the wide dayside sector, and highest values of the correlation coefficient are observed around local noon and in the Pc3 range. In this frequency band the correlation coefficient between the pulsation power and the SW speed has maximum values in the local morning. In addition, the frequency of selected events reveals a strong IMF control during closed magnetospheric conditions.

\section{Conclusions}

Although any comparison of experimental observations performed at different stations and at different times can be influenced by several factors such as the solar cycle, the seasonal variations, the selection criteria, the data processing, etc., the emerging overview of the present investigation suggests that several aspects of the pulsations activity at TNB may be significantly different than at lower latitudes. In particular a comparison with the results obtained in previous investigations allows drawing the following conclusions:

a. KHI, direct penetration of upstream waves, cusp proximity, substorm occurrence, etc., are all aspects which in general provide significant contributions to the pulsation power in the high-latitude magnetosphere. In this sense, the correspondence between the pulsation power and some external parameters (SW speed, IMF magnitude, cone angle) suggests that the relative contribution of exogenic sources at TNB may be more important than at somewhat smaller latitudes. In 

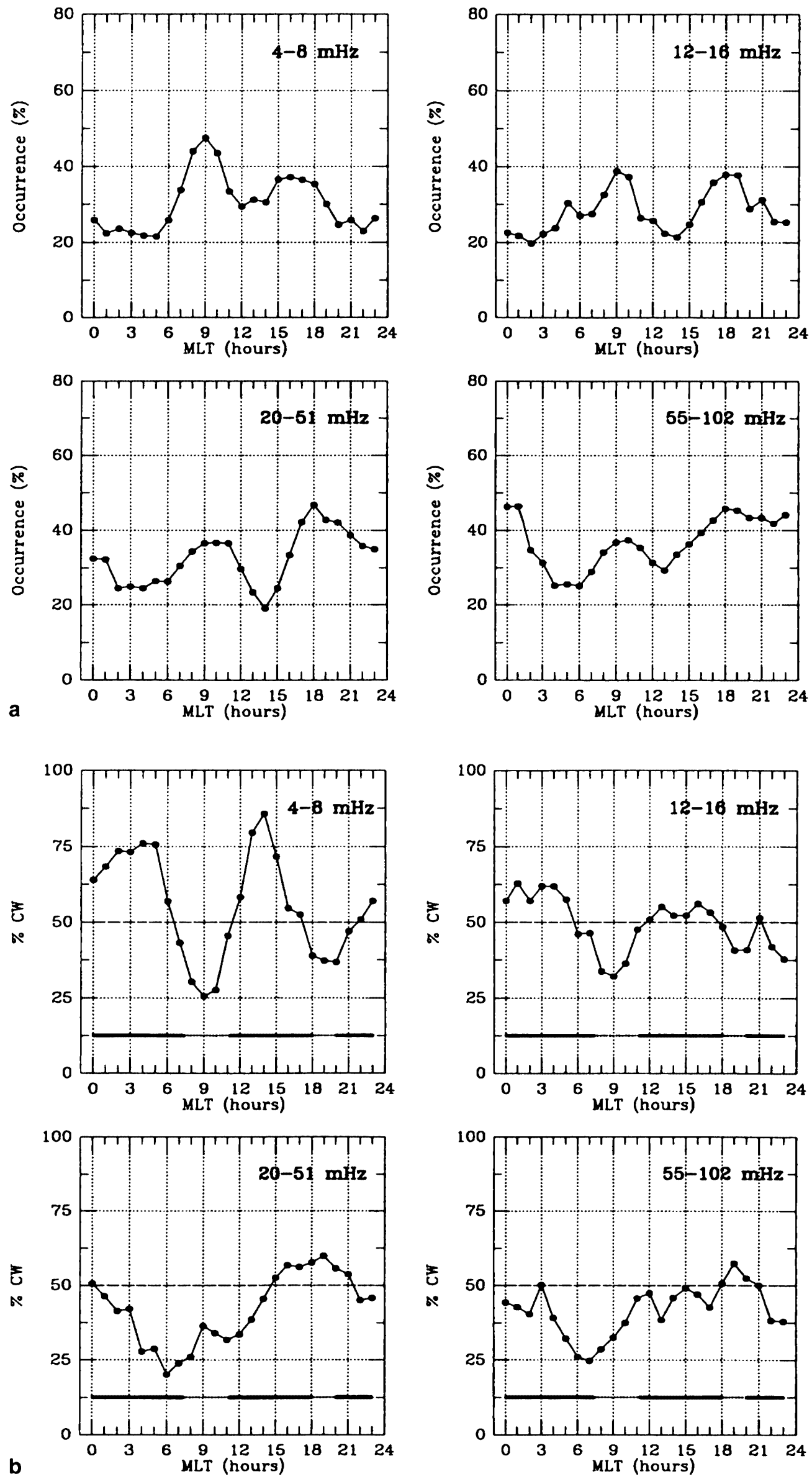

Fig. 7. a The daily variation of the percentage of polarized events for different frequency bands. b The daily variation of the percentage of $\mathrm{CW}$ polarized events. Bottom lines identify the time intervals in which $\mathrm{CW}$ (solid line) and CCW (dotted line) should be expected from the polarization pattern inferred by Samson et al. (1971) 
particular, the SW speed is clearly the key element in determining the level of the dayside pulsation power both in the Pc3 and Pc4 band, and the close correspondence between the level of the pulsation power and the geomagnetic activity simply reflects, in our opinion, the close relationship of both these parameters with the SW speed. In agreement with the results obtained in previous investigations at the same station (Villante et al., 1997, 1998; Lepidi et al., 1999), we would then conclude that, in general, the power level at $\sim 80^{\circ}$ is more strongly related to the SW speed than at smaller latitudes. Indeed, although the results of correlation analysis may be influenced by the different techniques and time scales, the results obtained at smaller latitudes suggest much smaller correlation coefficients between the pulsation power and the SW speed: for example, at South Pole, on an hourly time scale, Wolfe et al. (1987) found $r=0.42$ and $r=0.46$, in the Pc3 and Pc4 band, respectively, while Yumoto et al. (1987) estimated $r=0.64$ in the Pc3 band, after restricting the analysis in the noon hourly interval. The strong relationship between the pulsation power and the SW speed (which more explicitly emerges far from the noon sector, i.e. when the power level is less influenced by the cusp proximity) suggests in particular a dominant contribution of the KHI in the generation of surface waves and the amplification of already existing waves, as they are convected and transmitted through the high-latitude magnetopause (Yumoto et al., 1987).

b. At latitudes of the order of $74^{\circ}-75^{\circ}$ and in both hemispheres (Engebretson et al., 1986; Olson, 1986) the pulsation power showed peak values around magnetic noon (with maximum values typically encountered before noon) and a minor amplitude maximum in the nighttime hemisphere, which were attributed to the proximity of cusp and dayside aurora and to the nightside polar expansion of auroral substorms, respectively (Arnoldy et al., 1986; Engebretson et al., 1986): in this sense, the absence of the nighttime power enhancement at TNB might be reasonably interpreted in terms of a negligible substorm influence at $\sim 80^{\circ}$ on the Pc3 and Pc4 power. The results of recent investigations, although at lower frequencies, can be considered consistent with the present scenario: indeed, Lanzerotti et al. (1999), who examined geomagnetic fluctuations at $\sim 5 \mathrm{mHz}$, found dramatic differences between $75^{\circ}$ and $80^{\circ}$ and also remarked that the spectral features associated at $\sim 75^{\circ}$ with the southward turning of the IMF did not find correspondence at higher geomagnetic latitudes.

c. Some significant different features emerge in different bands. In particular, in the Pc4 band the occurrence of peaks of correlation between the pulsation power and the SW speed at the dawn and dusk meridian can be considered a new important signature in favour of a major role of the KHI along the magnetopause flanks. On the other hand, in agreement with present findings, Lepidi et al. (1999), who conducted a statistical analysis of low frequency fluctuations $(0.8-3.6 \mathrm{mHz})$ at TNB and McMurdo, found a very similar polarization pattern with four polarization reversals approximately at the same
MLTs. We would then conclude that, below $12 \mathrm{mHz}$, the experimental results are consistent with the polarization pattern predicted by theoretical models (Samson, 1972; Southwood, 1974; Chen and Hasegawa, 1974) for a resonant coupling between the fast compressional wave mode (generated at the magnetopause and propagating westward in the morning and eastward in the afternoon side of the Earth's magnetosphere) with the shear mode.

d. The additional cone angle control on the broad band pulsation power (which conflicts with the results obtained at South Pole for similar statistical analysis, Wolfe et al., 1987; Yumoto et al., 1987), the occurrence of maximum values of the correlation coefficient between the SW speed and the Pc3 power in the morning sector, and the strong IMF control of the frequency of selected events during closed magnetospheric conditions (it is interesting in this sense to remark that the regression coefficient is practically the same obtained at the same station during the passage of a high-velocity SW stream, Villante et al., 1999, and by Engebretson et al., 1986, for noon time observations at South Pole, and by Yumoto et al., 1984, at synchronous orbit) are all aspects which reveal that also the direct penetration of the external wave energy may be more important than at smaller latitudes (Engebretson et al., 1986, 1989). In this frequency range, the polarization pattern is less clear and opposite to that one which should be expected for antisunward propagating fluctuations: it represents a feature which, in our opinion, needs further investigations (also from a theoretical point of view) for a more definite understanding of all the aspects of high-latitude ULF pulsations.

Acknowledgements. This research activity at L'Aquila is supported by PNRA.

Topical Editor G. Chanteur thanks S. Vennerström and another referee for their help in evaluating this paper.

\section{References}

Arnoldy, R. L., L. J. Cahill Jr., and M. J. Engebretson, Review of hydromagnetic wave studies in the Antarctic, Rev. Geophys., 26, $1,1988$.

Bolshakova, O. V., and V. A. Troitskaya, The relation of the high latitude Pc3 intensity to the dayside cusp, Geomagn. Aeron. (English translation) 24, 663, 1984.

Chen, L., and A. Hasegawa, A theory of long period magnetic pulsations, 1, Steady state excitation of field line resonance, J. Geophys. Res., 79, 1024, 1974.

De Sanctis, Tesi di laurea, Università di L'Aquila, Italy, 1998.

Engebretson, M. J., B. J. Anderson, L. J. Cahill Jr., R. L. Arnoldy, P. T. Newell, C.-I. Meng, L. J. Zanetti, and A. T. Potemra, A multipoint case study of high latitude ULF pulsations, J. Geophys. Res., 94, 17 143, 1989.

Engebretson, M. J., B. J. Anderson, L. J. Cahill Jr., R. L. Arnoldy, T. J. Rosenberg, D. L. Carpenter, W. B. Gail, and R. H. Eather, Ionospheric signatures of cusp latitude Pc3 pulsations, J. Geophys. Res., 95, 2447, 1990.

Engebretson, M. J., J. R. Beck, R. L. Rairden, S. B. Mende, R. L. Arnoldy, L. J. Cahill Jr., and T. J. Rosenberg, Studies of the occurrence and properties of Pc3-4 magnetic field and auroral pulsations at South Pole, Antarctica, in Solar wind sources of magnetic ultra-low-frequency waves, Eds. Engebretson, M. J., K. Takahashi, and M. Scholer, AGU Geophys. Monog., 81, 345, 1994. 
Engebretson, M. J., K. H. Glassmaier, and M. Stellmark, The dependence of high latitude Pc5 wave power on solar wind velocity and on the phase of the high speed solar wind streams, J. Geophys. Res., 103, 26 271, 1998.

Engebretson, M. J., C.-I. Meng, R. L. Arnoldy, and L. J. Cahill Jr., Pc3 pulsations observed near the south polar cusp, J. Geophys. Res., 91, 8909, 1986.

Fowler, R. A., B. J. Kotick, and R. D. Elliot, Polarization analysis of natural and artificially induced geomagnetic micropulsations, J. Geophys Res., 72, 2871, 1967.

Fraser-Smith, A. C., ULF/lower ELF electromagnetic field measurements in the polar cap, Rev. Geophys., 20, 497, 1982.

Hughes, W. J., Pulsation research during the IMS, Rev. Geophys., 220, 641, 1982.

Lanzerotti, L. J., A. Shono, H. Fukunishi, and C. G. Maclennan, Long period hydromagnetic waves at very high geomagnetic latitudes, J. Geophys. Res., 104, 28 423, 1999.

Lepidi, S., P. Francia, U. Villante, L. J. Lanzerotti, and A. Meloni, Polarization pattern of low frequency geomagnetic field fluctuations $(0.8-3.6 \mathrm{mHz})$ at high and low latitude, J. Geophys. Res., 104, 305, 1999a.

Lepidi, S., P. Francia, U. Villante, A. Meloni, A. J. Lazarus, and R. L. Lepping, The Earth's passage of the April, 11, 1997 coronal ejecta: geomagnetic field fluctuations at high and low latitudes during northward interplanetary magnetic field conditions, Ann. Geophysicae, 17, 245, 1999 b.

Morris, R. J., and K. D. Cole, Pc3 magnetic pulsations at Davis, Antarctica, Planet. Space Sci., 35, 1437, 1987.

Olson, J. V., ULF signatures of the polar cusp, J. Geophys. Res., 91, $10055,1986$.

Olson, J. V., and C. A. L. Szuberla, A study of Pc3 coherence at high latitudes, J. Geophys. Res., 102, 11 375, 1997.

Samson, J. C., Three dimensional polarization characteristics of high latitude Pc5 geomagnetic micropulsations, J. Geophys. Res., 77, 6145, 1972.

Samson, J. C., J. A. Jacobs, and G. Rostoker, Latitude dependent characteristics of long period geomagnetic micropulsations, J. Geophys. Res., 76, 3675, 1971.
Szuberla, C. A. L., J. V. Olson, M. J. Engebretson, B. J. Fraser, S. Ables, and W. J. Huges, Interstation Pc3 coherence at cusp latitudes, Geophys. Res. Lett., 25, 2381, 1998.

Troitskaya, V., ULF wave investigations in the dayside cusp, $A d v$. Space Res., 5, 219, 1985.

Troistakaya, V., and O. V. Bolshakova, Diagnostics of the magnetosphere using multipoint measurements of ULF waves, Adv. Space Res., 8, 413, 1988.

Vellante, M., and U. Villante, Maximum entropy spectral analysis of artificial sinusoidal signals, J. Geophys. Res., 89, 351, 1984.

Vellante, M., U. Villante, M. De Lauretis, and P. Cerulli Irelli, An analysis of micropulsation events at a low latitude station during 1985, Planet. Space Sci., 37, 767, 1989.

Vennestrom, S., Dayside magnetic ULF power at high latitudes: a possible long term proxy for the solar wind velocity? J. Geophys. Res., 104, 10 145, 1999.

Villante, U., S. Lepidi, P. Francia, A. Meloni, and P. Palangio, Long period geomagnetic field fluctuations at Terra Nova Bay (Antarctica), Geophys. Res. Lett., 24, 1443, 1997.

Villante, U., P. Francia, S. Lepidi, M. De Lauretis, E. Pietropaolo, L. Cafarella, A. Meloni, A. J. Lazarus, R. P. Lepping, and F. Mariani, Geomagnetic field variations at low and high latitudes after the passage of the January 10-11, 1997 magnetic cloud, Geophys. Res. Lett., 25, 2593, 1998.

Villante, U., S. Lepidi, P. Francia, M. Vellante, A. Meloni, R. P. Lepping, and F. Mariani, Pc3 pulsations during variable IMF conditions, Ann. Geophysicae, 17, 490, 1999a.

Villante, U., S. Lepidi, P. Francia, M. Vellante, A. Meloni, and P. Palangio, ULF fluctuations at Terra Nova Bay (Antarctica), Ann. Geofis., (in press) 1999b.

Wolfe, A., E. Kamen, L. J. Lanzerotti, C. G. Maclennan, J. F. Bamber, and D. Venkatesan, ULF geomagnetic power at cusp latitudes in response to upstream solar wind conditions, J. Geophys. Res., 92, 168, 1987.

Yumoto, K. A., A. Wolfe, T. Teresawa, E. L. Kamen, and L. J. Lanzerotti, Dependence of Pc3 magnetic energy spectra at South Pole on upstream parameters, J. Geophys. Res., 92, 12 437, 1987. 